\title{
Visible 2-dimentional Photonic Crystal Laser
}

\author{
Zhaoyu Zhang ${ }^{1}$, Tomoyuki Yoshie ${ }^{2}$, Victor Liu ${ }^{1}$, Ting Hong ${ }^{1}$ and Axel Scherer ${ }^{1}$ \\ 1. Department of Electrical Engineering, California Institute of Technology, Pasadena, CA91125 \\ 2. Department of Electrical and Computer Engineering, Duke University, Durham, NC27708-0291 \\ Email: zhaoyu@caltech.edu Tel:626-395-2206Fax:626-683-9547.
}

\begin{abstract}
Visible 2-dimentional photonic crystal lasers were fabricated within membranes of InGaP/InGaAlP quantum well material emitting around $670 \mathrm{~nm}$. These red photonic crystal lasers with ultra-small mode volumes $(\sim 0.01 \mu \mathrm{m} 3)$ are ideally useful for spectroscopic sources. (C) 2007 Optical Society of America OCIS codes: (140.4780) Optical resonators; (140.5960) Semiconductor lasers; (140.7300) Visible lasers
\end{abstract}

Most research on photonic crystal lasers has so far focused on near-infrared wavelength emission using InGaAsP or InGaAs active material due to the applications in optical data communication [1-3]. Visible photonic crystal lasers operating in the spectral vicinity of $670 \mathrm{~nm}$ could enable a broad range of important applications, including high density optical recording and compact spectroscopic sources as ultrasmall sensors for biological and chemical detection.

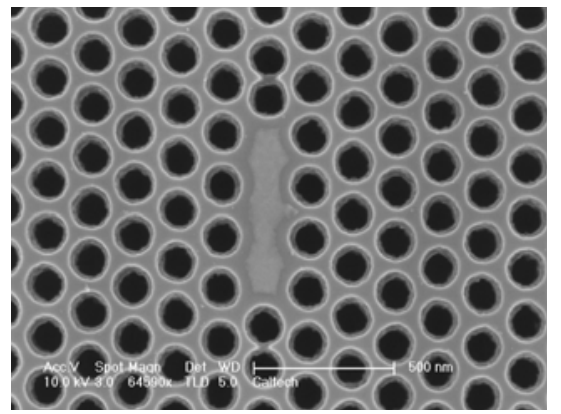

Figure 1. SEM micrographs of red photonic crystal laser cavity
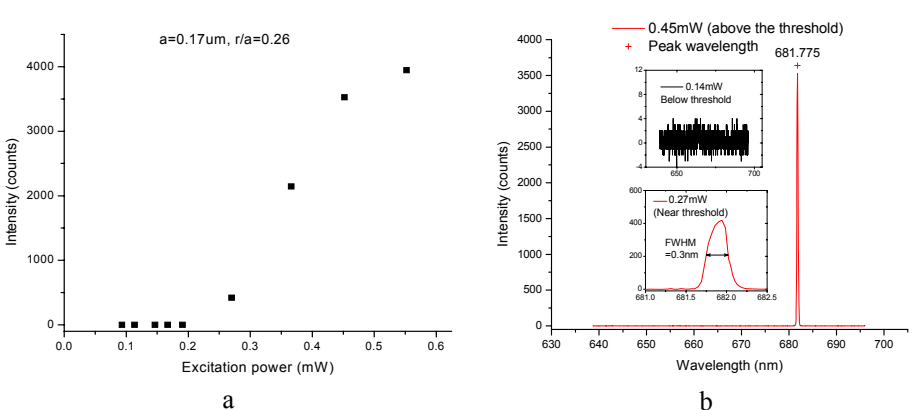

Figure 2. (a) L-L curve, (b)lasing spectra of a laser cavity with $\mathrm{a}=0.17 \mu \mathrm{m}$ and porosity factor $\mathrm{r} / \mathrm{a}=0.26$.

Photonic crystal slab structures were first grown by metallorganic chemical vapor deposition (MOCVD) of InGaP/InGaAlP quantum well material on top of sacrificial AlGaAs layers supported by GaAs substrates. Optical gain was provided by two $7 \mathrm{~nm}$ thick and compressively strained InGaP quantum wells which were separated by $10 \mathrm{~nm}$ InGaAlP barriers. The quantum well active material was placed in the center of a $180 \mathrm{~nm}$ thick InGaAlP slab, with a 700nm thick sacrificial AlGaAs layer between the slab and the GaAs substrate. The active quaternary material was designed to emit light at around $670 \mathrm{~nm}$. From the compressive strained quantum wells, light was strongly coupled into transverse electric (TE) modes. This epitaxially grown material was coated with a $100 \mathrm{~nm}$ SiON hard mask and $200 \mathrm{~nm}$ of Zep520 electron beam resist.

Electron beam lithography was then used to define the photonic crystal cavity pattern within the Zep520 resist. Reactive ion etching (RIE) was subsequently used to transfer the pattern from that resist into the SiON etch mask by using a CHF3 plasma. After removal of the resist, the hard mask pattern was further transferred through the active layer with an iodine-based inductively coupled plasma reactive ion etch (ICP-RIE). Time controlled oxidation of the AlGaAs by water vapor followed by the potassium hydroxide $(\mathrm{KOH})$ chemical dissolution of the aluminum oxide formed the suspended slab membranes as shown in Figure 1. Finally, diluted buffered HF was used to remove the SiON etch mask.

For this work, we used a high Q cavity design first proposed by Noda's group [4], consisting of a L3 linear defect within a triangular photonic crystal lattice of holes within a thin high-index slab as shown in Figure 1. We lithographically controlled the ratio between the hole shift and the lattice spacing to be 0.2 . Also, we lithographically varied the lattice spacing (a) within a range from $0.14 \mu \mathrm{m}-0.18 \mu \mathrm{m}$ and the porosity factor (r/a) from 0.25 to 0.29 . Fourteen periods of photonic crystal lattice of holes were used to surround the defect within the $170 \mathrm{~nm}$ thick quantum well active layer slab which has a refractive index of 
around 3.4 at $670 \mathrm{~nm}$. The cavities were optically pumped at room temperature using $5 \mathrm{~ns}$ pulses at $10 \mathrm{kHz}$ with a $408 \mathrm{~nm}$ InGaN diode laser. The pump beam was focused onto the sample to form a spot size about $2 \mu \mathrm{m}$ in diameter. The emission from the lasers was detected with a liquid nitrogen cooled CCD detector filtered by a monochromator which gives the spectral resolution of $\sim 0.1 \mathrm{~nm}$. Figure 2 shows luminescence spectra and the L(excitation power)-L(lasing peak intensity) curve from a device with lattice parameter $\mathrm{a}=170 \mathrm{~nm}$ and porosity factor $\mathrm{r} / \mathrm{a}=0.26$. The laser threshold was determined to be approximately $250 \mu \mathrm{W}$, and the linewidth was measured as $0.3 \mathrm{~nm}$ at threshold, yielding an effective Q of about 2000. Below threshold, only a broad background from the gain medium was measured from this cavity. Above $450 \mu \mathrm{W}$, heating of the laser cavity limited the output power, and the L-L curve saturated. The 2D photonic crystal cavity peak can be tuned by changing either the porosity or the lattice parameter of the photonic crystal lattice surrounding the nanocavity. The cavity exhibited distinct threshold and linearity in the output power above threshold but before saturation. The lasing characteristic was very sensitive to the position of the excitation beam spot ( $1 \mu \mathrm{m}$ movement), which indicates that lasing occurs from a localized defect mode.

Photonic crystal laser devices have been used in the past as refractive index sensors [5], indicating the refractive index of volumes as small as $10^{-17}$ liters, limited by the mode volume of 0.03 cubic microns at $1550 \mathrm{~nm}$ wavelength. Here, we have defined lasers within a wavelength range that is even more interesting for spectroscopic applications, as many of the fluorophores used for biological analysis are limited to the visible spectrum. We expect InGaP lasers to be very useful for biochemical analysis as well as for efficient displays and high-frequency lasers.

\section{REFERENCES}

[1] O. Painter, R. K. Lee, A. Scherer et al., "Two-dimensional photonic band-gap defect mode laser," Science 284 (5421), 18191821 (1999).

[2] M. Loncar, T. Yoshie, A. Scherer et al., "Low-threshold photonic crystal laser," Appl Phys Lett 81 (15), 2680-2682 (2002).

[3] T. Yoshie, O. B. Shchekin, H. Chen et al., "Quantum dot photonic crystal lasers," Electron Lett 38 (17), 967-968 (2002).

[4] Y. Akahane, T. Asano et al., "High-Q photonic nanocavity in a two-dimensional photonic crystal," Nature 425 (6961), 944-947 (2003)

[5] M. Loncar, A. Scherer, and Y. M. Qiu, "Photonic crystal laser sources for chemical detection," Appl Phys Lett 82 (26), 46484650 (2003) 